\title{
Cycles homoclinic to chaotic sets; robustness and resonance.
}

\author{
Peter Ashwin*
}

March 18, 1997

\begin{abstract}
For dynamical systems possessing invariant subspaces one can have a robust homoclinic cycle to a chaotic set. If such a cycle is stable, it manifests itself as long periods of quiescent chaotic behaviour interrupted by sudden transient 'bursts'. The time between the transients increases as the trajectory approaches the cycle. This behaviour for a cycle connecting symmetrically related chaotic sets has been called 'cycling chaos' by Dellnitz et al. (1995).

We characterise such cycles and their stability by means of normal Lyapunov exponents. We find persistence of states that are not Lyapunov stable but still attracting, and also states that are approximately periodic. For systems possessing a skew-product structure (such as naturally arises in chaotically forced systems) we show that the asymptotic stability and the attractivity of the cycle depends in a crucial way on what we call the footprint of the cycle. This is the spectrum of Lyapunov exponents of the chaotic invariant set in the expanding and contracting directions of the cycle. Numerical simulations and calculations for an example system of a homoclinic cycle parametrically forced by a Rössler attractor are presented; here we observe the creation of nearby chaotic attractors at resonance of transverse Lyapunov exponents.
\end{abstract}

Keywords: Homoclinic orbit, resonance, chaotic dynamics, chaotically forced system.

AMS (MOS): 34D45, 34C23, 58F12.

\section{Lead paragraph}

Dynamical systems generated by nonlinear ordinary differential equations can exhibit trajectories that are asymptotic to the same hyperbolic equilibrium as time limits to $\infty$ or $-\infty$. The union of the trajectory and the equilibrium is called a

\footnotetext{
${ }^{*}$ Institut Non Linéaire de Nice, 1361 Route des Lucioles, 06560 Valbonne, FRANCE. Permanent address: Department of Mathematical and Computing Sciences, University of Surrey, Guildford GU2 5XH, UK.
} 
homoclinic cycle and such cycles have been studied in great depth. Although the dynamics on the cycles are not very interesting (all points are either fixed or limit to fixed points as $t \rightarrow \pm \infty$ ), and moreover the cycle is typically destroyed on perturbation, they are found to be very important for the understanding of nearby dynamics, both in parameter and phase spaces. In particular, they provide a prototype of intermittent dynamical behaviour.

It has been recognised for several years that it is possible to have cycles to more complicated invariant sets, especially in systems with symmetry. Moreover, these cycles can be robust in that they are not destroyed by small perturbations (that preserve the symmetry). However, they have not been studied in much detail, partly because they can occur only in larger-dimensional systems.

In this paper we examine one of the simplest such cycles; a homoclinic cycle to a chaotic set. This would manifest itself in the behaviour of a nearby trajectory that stays close to a chaotic invariant set for long periods of time, interspersed with short intermittent bursts away into other regions of the phase space. The long periods may grow on average (if the cycle is attracting), have a mean period (if the cycle is not attracting) or just appear in transient behaviour.

We present a framework where it is possible to prove many results about such cycles; in particular we give criteria for robustness and attractivity of the cycle in terms of the transverse Lyapunov exponents of the invariant set. Moreover, we characterise a sort of 'blowout'-style bifurcation caused by resonance of certain Lyapunov exponents.

As an example, some numerical results from a chaotically forced variant of the Guckenheimer-Holmes robust homoclinic cycle are presented.

\section{Introduction}

Nonlinear dynamical systems with symmetry display a variety of exceptional behaviours due to the presence of subspaces forced to be dynamically invariant by the symmetry. In particular, homoclinic or heteroclinic cycles may be robust; these cycles provide interesting examples of non-ergodic attractors, cf. examples in $[27,29,28,14]$. Since the lengths of quiescent periods behave like a geometric progression, ergodic averages of attracted trajectories do not converge but continue to oscillate ad infinitum. In principle, heteroclinic cycles between any type of invariant set can occur, the case of equilibria being the best understood. As a trajectory progresses around the cycle it could pass near different invariant sets giving rise to a variety of different types of near-stationary dynamics.

What is surprising is that such cycles can arise in a robust way in several contexts, notably in systems with invariant subspaces. The existence and robustness of such cycles has been discussed in several recent papers, notably Melbourne et al. [18] and Dellnitz et al. [11]. Such behaviour has also been observed in a truncated model of Magnetoconvection (Rucklidge [26]). Although the reason why such cycles exist persistently in systems with symmetries is reasonably well understood, the actual dynamics and bifurcations are not. It is also possible for equilibria to be robustly 
connected by continua of connections $[18,8]$.

This paper examines the case of a homoclinic cycle to a chaotic set. Using normal Lyapunov exponents for chaotic sets in invariant subspaces and a skew product assumption we characterise the robustness and attractivity of the cycle. If there is a natural ergodic invariant measure for the chaotic set then this determines whether the set is attracting or not. However, the cycle may have a (locally) riddled basin; namely it can be attracting for a large measure nearby set, yet (locally) repel an open dense set.

At bifurcation from attracting to repelling cycles, there is a resonance $\lambda_{1}=-\lambda_{2}$ between normal Lyapunov exponents, and an unfolding of this will give rise to bifurcation of other attractors or repellors from the cycle. In the case of cycles between equilibria, these have the form of periodic solutions [9]. In the case we examine there is numerical evidence that they are chaotic attractors with have a well-defined mean time of lingering in a given neighbourhood of the invariant set. This time approaches infinity on nearing the bifurcation.

Section 2 gives some background, definitions and notation. A setting is presented where homoclinic cycles to chaotic sets occur in a robust way. Possible definitions of an attractor are also discussed along with their ergodic properties. Section 3 classifies the dynamics near cycles subject to an assumption of skew product structure of the flow near the cycle. By reference to a 'footprint' of Lyapunov exponents we give sufficient conditions that the cycle is asymptotically stable, a (Milnor) attractor or a chaotic saddle. We give sufficient conditions for local riddling of the basin of attraction of the cycle. We also investigate bifurcations that occur at resonance of such a cycle.

Using the theory from Section 3 we investigate in Section 4 an example vector field with $\mathbf{Z}_{2}^{3} \dot{+} \mathbf{Z}_{3}$ symmetry in $\mathbf{R}^{6}$ ( $A \dot{+} B$ is a semi-direct product of groups $A$ and $B)$. The vector field has a skew-product structure, i.e. it consists of a chaotic system (a Rössler attractor) parametrically forcing a robust homoclinic cycle. In section 5 we discuss the results in the context of the 'cycling chaos' of Dellnitz et al. [11] and the some of the assumptions made in Section 3.

\section{$2 \quad$ Setting and definitions}

The setting is similar to that in [6] with the difference that we consider flows rather than mappings. Let $M$ be a smooth $m$-manifold (we will work on a compact subset) with a Riemannian measure $\ell_{M}($.$) . We denote the tangent space to a manifold$ $N$ at $x \in N$ by $T_{x} N$. We assume the existence of smoothly embedded connected submanifolds $N$ and $P$ (of dimensions $n$ and $p$ respectively) such that $m>n>p>0$ and such that the following conditions are satisfied:

1. $P \subset \partial N$, i.e. $P$ is on the boundary of $N$.

2. For each $x \in P$ there is a neighbourhood $U$ in $N$ such that $U \cap N$ has (at least) two components, say $N_{1}$ and $N_{2}$. 
3. For $x \in P$ there exist $T_{1}(x)$ and $T_{2}(x)$ such that $T_{x} \bar{N}_{i}=T_{i} \oplus T_{x} P(i=1,2)$ and

$$
T_{x} M=T_{1} \oplus T_{2} \oplus T_{x} P .
$$

i.e. there is a splitting of $T_{x} M$. We assume that $T_{i}(x)$ are smooth in $x \in P$ and that the closures of the $N_{i}$ are also $n$-manifolds.

Figure 1 shows schematically the organisation of these invariant subspaces. We exclude the possibility that there are directions transverse to $T_{x} \bar{N}_{1} \oplus T_{x} \bar{N}_{2}$ although this is primarily to simplify the setting.

If this does not hold and even if there are unstable transverse directions, the results may still be valid (cf. the situation for heteroclinic cycles [19]). The conditions 1-3 ensure that flows respecting $N$ and $P$ can robustly have cycles in $N$ between invariant sets contained in $P$. Condition 3 implies that the stratification $(N, P)$ is Whitney regular [12], but is a much stronger requirement. We shall consider flows on $M$ generated by smooth $\left(C^{\infty}\right)$ vector fields that leave $N$ and $P$ invariant.

Definition 2.1 A smooth vector field $f \in X(M)$ is tangent to an embedded submanifold $N$ if $x \in N$ implies that $f(x) \in T_{x} N$.

Definition 2.2 We denote by $\tilde{X}(M)$ the set of all smooth vector fields tangent to both $N$ and $P$.

Suppose we have an ordinary differential equation on $M$

$$
\dot{x}=f(x)
$$

with $f \in \tilde{X}(M)$. Suppose that this generates a solution flow $\Phi: M \times \mathbf{R} \rightarrow M$. Clearly $\Phi(\cdot, t)$ leaves $P$ and $N$ invariant (we assume that the flow is defined for all $t \in \mathbf{R}$, although this is not essential for most of the results).

Remark 2.1 This invariant submanifold structure is typically part of a larger stratification of $M$ imposed by, for example, symmetries in the original equation. We shall ignore other strata, but note that it is important that there are no codimension-one strata in $N$ or $P$ which might form barriers to the flow.

\subsection{Stable sets and homoclinic cycles}

Suppose we have a flow-invariant compact set $A \subset M$. Such a set is said to be Lyapunov stable if for all neighbourhoods $U$ of $A$ there exists a neighbourhood $V$ of $A$ such that $\Phi(V, t) \subset U$ for all $t \in \mathbf{R}^{+}$. We define stable and unstable sets for $A$ :

$$
\begin{aligned}
& \mathcal{W}^{s}(A)=\{x \in M: \omega(x) \subset A\} \\
& \mathcal{W}^{u}(A)=\{x \in M: \alpha(x) \subset A\}
\end{aligned}
$$

where as usual

$$
\alpha(x)=\left\{\text { limits of sequences } \Phi\left(x, t_{i}\right) \text { with } t_{i} \rightarrow-\infty\right\}
$$




$$
\omega(x)=\left\{\text { limits of sequences } \Phi\left(x, t_{i}\right) \text { with } t_{i} \rightarrow \infty\right\} .
$$

Note that $\mathcal{W}^{s}(A)$ is also called the basin of attraction of $A$, e.g. [6]. These need not be manifolds but will be $\ell_{M}$-measurable subsets of $M$. If $A$ is Lyapunov stable and $\mathcal{W}^{s}(A)$ contains a neighbourhood of $A$, it is said to be asymptotically stable.

Definition 2.3 (Milnor [20]) $A$ compact invariant set $A$ is an attractor if $\ell_{M}\left(\mathcal{W}^{s}(A)\right)>$ 0 and any proper compact invariant $A^{\prime} \subset A$ has $\ell_{M}\left(\mathcal{W}^{s}\left(A^{\prime}\right)\right)<\ell_{M}\left(\mathcal{W}^{s}(A)\right)$. It is a minimal attractor if $\ell_{M}\left(\mathcal{W}^{s}\left(A^{\prime}\right)\right)=0$ for any such $A^{\prime}$.

If $A$ is not an attractor but $\mathcal{W}^{s}(A)$ is larger than $A$, it is said to be a saddle. Note that we do not assume that $A$ is transitive.

Definition 2.4 There is a homoclinic connection $C(A)$ to the chaotic set $A$ if

$$
C(A)=\mathcal{W}^{u}(A) \cap \mathcal{W}^{s}(A) \backslash A
$$

is non-empty. The set $C(A) \cup A$ is a homoclinic cycle.

In the case of $A$ being an attractor but not Lyapunov stable, it may have a locally riddled basin in the following sense:

Definition 2.5 (cf [6]) An attractor $A$ has a locally riddled basin if there exists an neighbourhood $V$ containing $A$ such that for any open set $U$ that intersects $A$,

$$
\ell_{M}\left(U \cap \bigcup_{t \in \mathbf{R}^{+}, x \in V^{c}} \Phi(x,-t)\right)>0 .
$$

This means that arbitrarily close to all points in $A$ there are positive measure sets that depart from a fixed neighbourhood $V$ of $A .\left(V^{c}\right.$ denotes the complement of $V$ in $M$ )

\subsection{Generic sets for measures}

The measures we consider are all Borel measures, and all invariant measures we consider are probability measures; i.e. normalised to $\mu(M)=1$. Define

$$
\mathcal{M}(A)=\{\Phi \text {-invariant probability measures with } \operatorname{supp}(\mu) \subset A\}
$$

and the subset of ergodic invariant measures $\operatorname{Erg}(A)$. We say a compact invariant set $A$ is chaotic if $\operatorname{Erg}(A)$ is not a finite set. For any $\mu \in \operatorname{Erg}(A)$ we define $\mathcal{G}^{+}$the forward and $\mathcal{G}^{-}$the backward generic set thus:

$\mathcal{G}^{ \pm}(\mu)=\left\{x \in M: \lim _{T \rightarrow \infty} \frac{1}{T} \int_{t=0}^{T} F(\Phi(x, \pm t))=\int F(y) d \mu(y) \quad\right.$ for all $\left.F \in C^{0}(M, \mathbf{R})\right\}$.

The sets $\mathcal{G}^{ \pm}$are easily seen to be invariant under the flow $\Phi(\cdot, t)$ for $t \in \mathbf{R}$. Birkhoff's ergodic theorem implies that $\mu\left(\mathcal{G}^{ \pm}(\mu)\right)=\mu\left(\mathcal{G}^{-}(\mu)\right)=1$; however, these generic sets may be much larger than a set of full $\mu$-measure (though they may be very small in a topological sense; see Sigmund [28]). 
Definition 2.6 (e.g. [27]) An attractor $A$ has a natural measure $\mu_{n}$ if there is a unique ergodic invariant measure $\mu_{n}$ with support contained in $A$ and

$$
\ell_{M}\left(\mathcal{G}^{+}\left(\mu_{n}\right)\right)>0 \text {. }
$$

There are measurable subsets of the stable and unstable sets; given $A$ and $\mu \in$ $\operatorname{Erg}(A)$ define

$$
\begin{aligned}
& \mathcal{W}^{s}\left(A, \mu_{n}\right)=\mathcal{W}^{s}(A) \cap \mathcal{G}^{+}\left(\mu_{n}\right), \\
& \mathcal{W}^{u}\left(A, \mu_{n}\right)=\mathcal{W}^{u}(A) \cap \mathcal{G}^{-}\left(\mu_{n}\right)
\end{aligned}
$$

(note that $\mathcal{G}^{+}\left(\mu_{n}\right)$ is not necessarily contained in $\mathcal{W}^{s}(A)$; especially for attracting homoclinic orbits where $\ell_{M}\left(\mathcal{G}^{+}\right)>0$ but $\ell_{M}\left(\mathcal{W}^{s}\right)=0$.) Similarly define the subset of $C(A)$ :

$$
C\left(A, \mu_{n}\right)=\mathcal{W}^{u}\left(A, \mu_{n}\right) \cap \mathcal{W}^{u}\left(A, \mu_{n}\right)
$$

whose averages are generic for the natural measure $\mu_{n}$. Note that there do exist flows with an attractor $C$ such that for all $\mu \in \operatorname{Erg}(C)$ we have $\ell_{M}\left(\mathcal{W}^{s}(\operatorname{supp}(\mu))\right)=0$. This can be seen in flows with attracting homoclinic or heteroclinic orbits to fixed points, or at blowout bifurcation [7]. For a discussion of genericity and time averages, see Sigmund [28]. We call a compact invariant set $A$ regular if for all $\mu \in \operatorname{Erg}(A)$ we have

$$
\ell_{M}\left(\mathcal{W}^{s}(A, \mu)\right) \cdot \ell_{M}\left(\mathcal{W}^{u}(A, \mu)\right)=0 .
$$

Otherwise we say it is irregular. We conjecture that a large (if not generic) set of smooth dynamical systems have regular invariant sets. For one-parameter systems one can robustly find irregular sets (for example, a saddle-node on a circle), but note that examples of attractors $A$ with riddled basins in $[1,4]$ are regular.

A natural measure $\mu_{n}$ is called a Sinai-Bowen-Ruelle measure if it is absolutely continuous on unstable manifolds. By e.g. [24, 23] it follows that an attractor supporting a Sinai-Ruelle-Bowen measure is regular: this is because of absolute continuity of the stable and unstable foliations.

Finally, we will be interested primarily in clean homoclinic connections:

Definition 2.7 A homoclinic connection $C(A)$ is clean if

1. $\mathcal{W}^{u}(A) \subset \mathcal{W}^{s}(A)$, implying that $C(A)=\mathcal{W}^{u}(A)$.

2. There is a smooth codimension-one section to the flow that intersects a neighbourhood of $C(A)$.

Otherwise, the connection is dirty.

Normal Lyapunov exponents (see also [1, 6])

Given an initial point $x \in M$ and $v \in T_{x} M$ the Lyapunov exponent $\lambda(x, v)$ for the flow $\Phi$ is defined by

$$
\lambda(x, v)=\lim _{t \rightarrow \infty} \frac{1}{n} \log \left|d_{x} \Phi(x, t) v\right|
$$


$\left(d_{x}\right.$ indicates the derivative with respect to $\left.x\right)$ if this limit exists. The Multiplicative ergodic theorem of Oseledec [21] implies that for a given ergodic invariant measure $\mu$, for $\mu$-a.a. $x$ and all $v$ this limit exists and takes on finitely many values given by a $\mu$-measurable splitting of $T_{x} M$. As we are considering a flow, there will always be one Lyapunov exponent that is zero due to time-translation invariance.

If a chaotic Lyapunov-stable attractor $A$ is contained in the embedded submanifold $P$ we can, for each $\mu \in \operatorname{Erg}(A)$ define a set of $m$ Lyapunov exponents (with multiplicity) and divide these into two groups consisting of $p$ tangential and $m-p$ normal Lyapunov exponents. These exist for a set of points in $A$ with full $\mu$-measure.

By restricting to small enough $\delta>0$ and using (1), for any compact $W \subset P$ containing $A$ there is a diffeomorphism between

$$
N^{\delta}=\left\{\left(v_{1}, v_{2}, u\right) \in T_{1} \oplus T_{2} \oplus W: \max \left(\left\|v_{i}\right\|, d(u, A)\right) \leq \delta\right\}
$$

and a neighbourhood of $W$ given by the exponential map. Therefore we work in the two spaces interchangeably and write

$$
x=\left(v_{1}, v_{2}, u\right)
$$

in such a neighbourhood.

The invariance of the subspaces $N_{i}$ mean that for any $f \in \tilde{X}(M)$ we can write $\dot{x}=f(x)$ in $N^{\delta}$ as

$$
\begin{aligned}
\dot{v}_{1} & =a_{1}\left(v_{1}, v_{2}, u\right) v_{1} \\
\dot{v}_{2} & =a_{2}\left(v_{1}, v_{2}, u\right) v_{2} \\
\dot{u} & =b\left(v_{1}, v_{2}, u\right) .
\end{aligned}
$$

with $a_{i}$ and $b$ smooth in all their arguments; $a_{i}$ are $(n-p)^{2}$ matrices.

This can be linearised near a trajectory of the flow $p(t) \in P, u=p(t)+v_{p}$ to give

$$
\begin{aligned}
& \dot{v}_{1}=a_{1}(0,0, p) v_{1} \\
& \dot{v}_{2}=a_{2}(0,0, p) v_{2} \\
& \dot{v}_{p}=\beta_{1}(p) v_{1}+\beta_{2}(p) v_{2}+\beta_{3}(p) v_{p} .
\end{aligned}
$$

The solution flow of this linearised ODE will have the form

$$
\begin{aligned}
& v_{1}(t)=\Psi_{1}\left(p_{0}, t\right) v_{1} \\
& v_{2}(t)=\Psi_{2}\left(p_{0}, t\right) v_{2} \\
& v_{p}(t)=\Psi_{4}\left(p_{0}, t\right) v_{1}+\Psi_{5}\left(p_{0}, t\right) v_{2}+\Psi_{3}\left(p_{0}, t\right) v_{p} .
\end{aligned}
$$

where the $\Psi_{i}\left(p_{0}, t\right)$ are linear maps that are appropriate derivatives of $\Phi$ evaluated along the trajectory $p(t)=\Phi\left(p_{0}, t\right)$.

The normal Lyapunov exponents correspond to the Lyapunov exponents of the matrix skew product flows $\Psi_{i}(i=1,2)$ and accordingly can be split into two groups

$$
\begin{cases}\lambda_{1}(\mu) & \text { corresponding to } \Psi_{1} \\ \lambda_{2}(\mu) & \text { corresponding to } \Psi_{2}\end{cases}
$$


More precisely, the normal Lyapunov exponents $\lambda_{i}$ are characteristic exponents of the matrix cocyles $\Psi_{i}$. For $\mu$-a.a. $p_{0} \in P$ and all $v \in N_{i}$ with $|v|=1$ they are given by

$$
\lambda_{i}\left(p_{0}, v\right)=\lim _{t \rightarrow \infty} \frac{1}{t} \log \left|\Psi_{i}\left(p_{0}, t\right) v\right| .
$$

We now adapt the setting of [6] to this context. We define

$$
\begin{aligned}
& \bar{\lambda}_{i}(\mu)=\max \lambda_{i}(\mu) \\
& \underline{\lambda}_{i}(\mu)=\min \lambda_{i}(\mu) \\
& \quad \text { and } \\
& \lambda_{i}^{\max }=\sup _{\mu \in \operatorname{Erg}(A)} \bar{\lambda}_{i}(\mu) \\
& \lambda_{i}^{\min }=\inf _{\mu \in \operatorname{Erg}(A)} \underline{\lambda}_{i}(\mu) .
\end{aligned}
$$

Since the flow is invertible, the maximum and minimum Lyapunov exponents are finite.

Define the matrix norm $\|M\|=\max _{|v|=1}\|M v\|$ and note that for $|v| \neq 0$

$$
\frac{1}{\left\|\Psi_{i}^{-1}\left(p_{0}, t\right)\right\|} \leq \frac{\left|\Psi_{i}\left(p_{0}, t\right) v\right|}{|v|} \leq\left\|\Psi_{i}\left(p_{0}, t\right)\right\| .
$$

It follows that

$$
\lambda_{i}^{\max }=\sup _{p_{0} \in A} \lim _{t \rightarrow \infty} \frac{1}{t} \log \left\|\Psi_{i}\left(p_{0}, t\right)\right\| \quad \text { and } \quad \lambda_{i}^{\min }=\sup _{p_{0} \in A} \lim _{t \rightarrow \infty}-\frac{1}{t} \log \left\|\Psi_{i}^{-1}\left(p_{0}, t\right)\right\| .
$$

These imply that for all $\epsilon>0$ with $\lambda_{i}>\epsilon$ there exist $T$ such that

$$
\begin{aligned}
\exp \left(\left(\lambda_{i}^{\max }-\epsilon\right) t\right) & <\sup _{p_{0} \in A}\left\|\Psi_{i}\left(p_{0}, t\right)\right\|<\exp \left(\left(\lambda_{i}^{\max }+\epsilon\right) t\right) \\
\exp \left(\left(-\lambda_{i}^{\min }-\epsilon\right) t\right) & <\sup _{p_{0} \in A}\left\|\Psi_{i}^{-1}\left(p_{0}, t\right)\right\|<\exp \left(\left(-\lambda_{i}^{\min }+\epsilon\right) t\right)
\end{aligned}
$$

for all $t>T$. Moreover, for a given $\mu \in \operatorname{Erg}(A)$ and setting $\Lambda_{i}(\mu)=\max \left(\lambda_{i}(\mu)\right)$ there is a full measure set of $\left(v_{i}, p_{0}\right)$ (in the product of Lebesgue measure with $\mu$ ) and a measurable $T\left(v_{i}, p_{0}\right)>0$ such that in this subset

$$
\exp \left(\left(\bar{\lambda}_{i}(\mu)-\epsilon\right) t\right)<\frac{\left|\Psi_{i}\left(p_{0}, t\right) v_{i}\right|}{\left|v_{i}\right|}<\exp \left(\left(\bar{\lambda}_{i}(\mu)+\epsilon\right) t\right)
$$

for all $t \geq T$. (NB $\underline{\lambda}_{i}(\mu)$ determines the growth of $\mu$-almost all trajectories as $t \rightarrow-\infty$.)

Modification to nonlinear cocycle flows We can rewrite the nonlinear ODE (2) in the form

$$
\begin{aligned}
\dot{v}_{1} & =\left(a_{1}(0,0, u)+O\left(\left|v_{1}\right|+\left|v_{2}\right|\right)\right) v_{1} \\
\dot{v}_{2} & =\left(a_{2}(0,0, u)+O\left(\left|v_{1}\right|+\left|v_{2}\right|\right)\right) v_{2} \\
\dot{u} & =b\left(v_{1}, v_{2}, u\right) .
\end{aligned}
$$

where the estimates $O\left(\left|v_{1}\right|,\left|v_{2}\right|\right)$ are uniform in $u$. By restricting to a neighbourhood with $\max \left(\left|v_{1}\right|,\left|v_{2}\right|\right)$ small enough that $\left\|A_{1}\right\|<\epsilon / 2$ we can obtain the same bounds as in (7) on replacing $\Psi_{1}$ by the first component of the nonlinear cocyle $\Phi\left(v_{1}, v_{2}, p, t\right)$. 
The footprint of Lyapunov exponents In the following section, we see that the stability and bifurcation of structurally stable cycles between chaotic sets in this setup depends in a fundamental way on the footprint of A defined as the subset of the plane:

$$
S(A)=\left\{\left(\nu_{1}, \nu_{2}\right): \nu_{i} \in \lambda_{i}\left(\mu_{i}\right) \text { for some } \mu_{i} \in \operatorname{Erg}(A)\right\}
$$

If there is a natural measure $\mu_{n}$ we define

$$
\left.\left.s_{n}=\left(\lambda_{1}^{n}, \lambda_{2}^{n}\right)=\left(\bar{\lambda}_{1}\left(\mu_{n}\right)\right), \bar{\lambda}_{2}\left(\mu_{n}\right)\right)\right)
$$

Note that $\left(\lambda_{1}^{n}, \lambda_{2}^{n}\right) \in S(A)$. By [6, Thm. 3.5], if $A$ is Axiom-A then it is possible to show that in a certain sense, it is generically contained in the interior of the convex hull of $S(A)$.

Rates of approach and slowing down Suppose that $C(A) \cup A$ is a homoclinic cycle to a chaotic invariant set and $\Sigma$ is a local section that is transverse to the flow and to $\mathcal{W}^{s}(A)$. We fix on a particular trajectory that is assumed to intersect $\Sigma$ infinitely often. Let $m_{k}=\gamma\left(t_{k}\right) \in \Sigma$ be the $k$ th crossing of $\Sigma$ and let $d_{k}=$ $d\left(m_{k}, \mathcal{W}^{s}(A)\right)$.

Definition 2.8 The rate of approach is defined to be

$$
R(k)=\frac{\log d_{k+1}}{\log d_{k}}
$$

The rate of slowing down is defined to be

$$
r(k)=\frac{t_{k+1}-t_{k}}{t_{k}-t_{k-1}}
$$

The asymptotic rate of approach is defined to be $R=\lim _{k \rightarrow \infty} R(k)$ (if the limit exists). The asymptotic rate of slowing down $r$ is defined similarly.

If $A$ is an equilibrium and $C(A)$ an asymptotically stable homoclinic connection then $R(k)$ and $r(k)$ will converge to the same constant; namely the ratio of the two leading eigenvalues.

\subsection{Robustness}

The fact that $C(A)$ can be robust to perturbations preserving the invariance of $N$ and $P$ is demonstrated by the following proposition. This result is not optimal, in particular it is probably possible to weaken assumption (3) to $\lambda_{1}^{\max }<0<\lambda_{2}^{\min }$.

Proposition 2.1 Suppose for the flow of $\dot{x}=f(x)$

1. There is a clean homoclinic connection $C(A) \subset N$. 
2. The set $A$ is an attractor for the restriction to $P$, upper semi-continuous in $X(P)$ (this follows e.g. if $A$ is asymptotically stable for the flow restricted to $P)$.

3. There is a compact neighbourhood $W$ of $A$ in $P$ such that

$$
\left\|\exp \left(a_{1}\left(0,0, p_{0}\right)\right)\right\|<1<\left\|\exp \left(-a_{2}\left(0,0, p_{0}\right)\right)\right\|^{-1}
$$

for all $p_{0} \in W$, i.e. local uniform contraction in $N_{1}$ and local uniform expansion in $N_{2}$.

Then there is a neighbourhood of $f$ in $\tilde{X}(M)$ with homoclinic cycles to invariant sets in $P$.

\section{Proof :}

The upper-semicontinuity of $A$ implies that given any neighbourhood $B$ (in $P$ ) of $A$, there is a neighbourhood $F$ of $f$ possessing attractors $A^{\prime}$ contained in $B$. Moreover, because

$$
\left\|\Psi_{1}\left(p_{0}, t\right)\right\|<\exp (t \rho)
$$

for some $\rho<0$ condition (3) implies that for all $p_{0}$ in a neighbourhood of $A$ and all $t>0$ we have

$$
\left\|\Psi_{1}\left(p_{0}, t\right)\right\|<1<\frac{1}{\left\|\Psi_{2}^{-1}\left(p_{0}, t\right)\right\|} .
$$

This is a robust property, i.e. it holds in a neighbourhood $F$ of $f$ in $\tilde{X}(M)$. The assumption that $C(A)$ is clean means we can find a smooth section to the flow which we can transport forwards and backwards to give sections to the flow $\Sigma_{i}$ transverse to $N_{i}$ such that $\Sigma_{2} \cap \mathcal{W}^{u}(A)$ is non-empty. A neighbourhood of $\Sigma_{2} \cap \mathcal{W}^{u}(A)$ is transported to $\Sigma_{1}$ robustly, and the local contraction and expansion imply there will be clean homoclinic connections $C\left(A^{\prime}\right)$ in all nearby systems (of course, $A$ may collapse to a periodic orbit $A^{\prime}$ on perturbation).

\section{Classification of cycles homoclinic to chaotic sets}

In this section we classify the dynamics near a homoclinic cycle to a chaotic set by reference to its footprint (9). This classification just relies on there being a robust connection in $N$ and some properties of the linearisation of the flow near $A$. We partition the plane into the union of six regions, i.e.

$$
\mathbf{R}^{2}=\bigcup_{i=1}^{6} \bar{U}_{i}
$$


where

$$
\begin{aligned}
& U_{1}=\left\{\left(\lambda_{1}, \lambda_{2}\right): \lambda_{1}>0 \text { and } \lambda_{2}>0\right\} \\
& U_{2}=\left\{\left(\lambda_{1}, \lambda_{2}\right):-\lambda_{2}<\lambda_{1}<0\right\} \\
& U_{3}=\left\{\left(\lambda_{1}, \lambda_{2}\right):-\lambda_{1}<\lambda_{2}<0\right\} \\
& U_{4}=\left\{\left(\lambda_{1}, \lambda_{2}\right): \text { and }-\lambda_{1}>\lambda_{2}>0\right\} \\
& U_{5}=\left\{\left(\lambda_{1}, \lambda_{2}\right):-\lambda_{2}>\lambda_{1}>0\right\} \\
& U_{6}=\left\{\left(\lambda_{1}, \lambda_{2}\right): \lambda_{1}<0 \text { and } \lambda_{2}<0\right\} .
\end{aligned}
$$

We also define the union of the $\lambda_{1}$ and $\lambda_{2}$ axes:

$$
V=\left\{\left(\lambda_{1}, \lambda_{2}\right): \lambda_{1} \lambda_{2}=0\right\}
$$

This partition is shown in Figure 2. The relation of the set $S(A)$ and $s_{n}$ to this partition determines the dynamics of the system. Firstly, we classify cases with asymptotic stability. We denote the bounding rectangle $S(A)$ (precisely the convex hull of $S(A))$ by

$$
\operatorname{Box}(S(A))=\left[\lambda_{1}^{\min }, \lambda_{1}^{\max }\right] \times\left[\lambda_{2}^{\min }, \lambda_{2}^{\max }\right]
$$

Classification for skew product systems We make the simplifying assumption that the system takes the form of a skew product over the flow on $P$. This means that we have a smooth factorising map $\Pi: M \rightarrow P$ given by a projection onto $P$ such that for all $t$ the following diagram commutes.

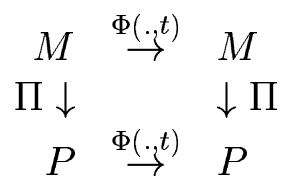

This implies that $\Psi_{4}=\Psi_{5}=0$ in (4).

Theorem 3.1 Suppose we have a clean homoclinic cycle $C(A)$ to a chaotic set $A$ and the flow on a neighbourhood of $N$ is a skew product over the flow on $P$. We classify the asymptotic stability of $A$ and $C$ according to the following list:

If $\operatorname{Box}(S(A)) \subset U_{1}$ then $A$ is normally repelling.

If $\operatorname{Box}(S(A)) \subset U_{2} \cup U_{3}$ then $C(A)$ is normally repelling.

If $\operatorname{Box}(S(A)) \subset U_{4} \cup U_{5}$ then $C(A)$ is asymptotically stable.

If $\operatorname{Box}(S(A)) \subset U_{6}$ then $A$ is asymptotically stable.

A 'measurable' version of this theorem is as follows.

Theorem 3.2 Assume we have a chaotic set $A$ with a natural measure $\mu_{n}$ such that $\mathcal{W}^{s}\left(\mu_{n}\right)$ has full measure in some neighbourhood of $A$ in $P$. Suppose we have a clean homoclinic cycle $C(A)$ and that the flow on a neighbourhood of $N$ is a skew product over the flow on $P$. We classify the stability of $A$ and $C(A)$ according to the following list: 
If $s_{n} \notin U_{6}$ then $A$ is a chaotic saddle.

If $s_{n} \in U_{2} \cup U_{3}$ and $\operatorname{Box}(S(A)) \cap V=\emptyset$ then $C(A)$ is not an attractor.

If $s_{n} \in U_{4} \cup U_{5}$ and $\operatorname{Box}(S(A)) \cap V=\emptyset$ then $C(A)$ is a (Milnor) attractor.

If $s_{n} \in U_{6}$ then $A$ is a (Milnor) attractor.

Observe that there is a unique natural measure supported on $A$ because of the full measure neighbourhood assumption. The remainder of this section consists of proofs of Theorems 3.1 and 3.2. We introduce sections on $\partial N^{\delta}$ to the flow at

$$
\Sigma_{i}^{\delta, \gamma}=\left\{\left(v_{1}, v_{2}, u\right) \in N^{\delta}:\left\|v_{1}\right\|=\delta,\left\|v_{2}\right\|<\gamma\right\}
$$

for $0<\gamma<\delta$. These transversely intersect $N_{1}$ and $N_{2}$ near $A$; see figure 3 . On these sections, we can define maps from a domain $\mathcal{D}_{i j} \subset \Sigma_{i}^{\delta, \gamma}$ thus;

$$
\Psi_{i j}: D_{i j} \rightarrow \Sigma_{j}^{\delta, \gamma}
$$

given by the first passage: i.e. we define

$$
T_{i j}: \Sigma_{i} \rightarrow \mathbf{R}^{+} \cup\{+\infty\}
$$

as the infimum of all $t>0$ such that $\Phi(x, t) \in \Sigma_{j}$, and then

$$
\mathcal{D}_{i j}=\left\{x \in \Sigma_{i}: T_{i j}<\infty\right\}
$$

and for $x \in \mathcal{D}_{i j}$

$$
\Psi_{i j}(x)=\Phi\left(x, T_{i j}(x)\right) .
$$

In the case that $T_{i j}(x)=\infty$ the map is not defined. Without loss of generality, we only examine cycles that depart from $A$ in the $N_{2}$ direction and approach it in the $N_{1}$ direction.

Approximation of $\Psi_{21}$ : 'far returns' We consider the evolution from $\Sigma_{2}^{\delta, \gamma}$ to $\Sigma_{1}^{\delta, \gamma}$. This interchanges the spaces $N_{1}$ and $N_{2}$ and leads to a map (where defined) of the form:

$$
\Psi_{21}\left(v_{1}, v_{2}, u\right)=\left(T_{1}\left(u, t_{2}\right) e^{O\left(\left|v_{i}\right| t_{2}\right)} v_{2}, T_{2}\left(u, t_{2}\right) e^{O\left(\left|v_{i}\right| t_{2}\right)} v_{1}, \Phi\left(u, t_{2}\right)\right)
$$

where $t_{2}=T_{21}\left(v_{1}, v_{2}, u\right)=T_{21}(u)+O\left(\left|v_{i}\right|\right)$. The $T_{i}$ are continuous matrix-valued functions of $u$ and $t_{2}$. This map is well-defined and the error-bounds uniform if we restrict to a domain

$$
\mathcal{D}_{2}^{\rho}=\left\{x \in \mathcal{D}_{21}: t_{2}(x)<\rho\right\} \subset \Sigma_{2}
$$

for given $\rho<\infty$. Since the cycle is clean, there is a $\rho<\infty$ such that $\mathcal{D}_{2}^{\rho}$ contains a neighbourhood of the unstable manifold in $\Sigma_{2}$, and so the error terms are uniform in this neighbourhood. 
Approximation of $\Psi_{12}$ : 'near returns' Given an initial point $\left(v_{1}, v_{2}, u\right)$ we linearise about the flow of $(0,0, u)$. For small enough $\delta$ and $\left(v_{1}, v_{2}, u\right) \in N^{\delta}$ note that, where defined, we have

$$
\Psi_{12}\left(v_{1}, v_{2}, u\right)=\Phi\left(u, t_{1}\right)+d \Phi\left(u, t_{1}\right)\left(v_{1}, v_{2}, 0\right) e^{O\left(\left|v_{i}\right| t_{1}\right)}
$$

where $t_{1}=T_{12}\left(v_{1}, v_{2}, u\right)$. From (4) we can write

$$
\Psi_{12}\left(v_{1}, v_{2}, u\right)=\left(\Psi_{1}\left(u, t_{1}\right) e^{O\left(\left|v_{i}\right| t_{1}\right)} v_{1}, \Psi_{2}\left(u, t_{1}\right) e^{O\left(\left|v_{i}\right| t_{1}\right)} v_{2}, \Phi\left(u, t_{1}\right)\right)
$$

where similarly, the error terms are uniform if we restrict to $\left(v_{1}, v_{2}, u\right)$ such that $t_{1}<\rho<\infty$. We similarly define

$$
\mathcal{D}_{1}^{\rho}=\left\{x \in \mathcal{D}_{12}: t_{1}(x)<\rho\right\}
$$

The return map Defining $F=\Psi_{21} \circ \Psi_{12}$ we obtain a map from a subset of the surface of section $\Sigma_{1}^{\delta}$ to itself that (where defined) has the form:

$$
F\left(v_{1}, v_{2}, u\right)=\left(v_{1}^{\prime}, v_{2}^{\prime}, u^{\prime}\right)
$$

with

$$
\begin{aligned}
& v_{1}^{\prime}=T_{1}\left(\Phi\left(u, t_{1}\right), t_{2}\right) \Psi_{2}\left(u, t_{1}\right) e^{O\left(\left|v_{i}\right|\left(t_{1}+t_{2}\right)\right)} v_{2} \\
& v_{2}^{\prime}=T_{2}\left(\Phi\left(u, t_{1}\right), t_{2}\right) \Psi_{1}\left(u, t_{1}\right) e^{O\left(\left|v_{i}\right|\left(t_{1}+t_{2}\right)\right)} v_{1} \\
& u^{\prime}=\Phi\left(u, t_{1}+t_{2}\right)
\end{aligned}
$$

Since we are interested in the exponential rates of growth or shrinkage of $\left|v_{2}\right|$ on iteration, we can effectively ignore the exponential error terms by limiting to small enough $\delta$ and using Section 2.2.

\subsection{Asymptotic stability}

Lemma 3.1 If $\operatorname{Box}(S(A)) \subset U_{6}$ then $A$ is an asymptotically stable attractor; if $S(A) \subset U_{1}$ then it is a normal repellor. In both cases $C(A)$ is empty.

\section{Proof :}

In first case $\lambda_{i}^{\max }<0$ for $i=1,2$ and so we can apply [6, Thm. 2.12] to give the result. In the second case $\lambda_{i}^{\text {min }}>0$ for $i=1,2$ and the same theorem can be applied.

Consider the clean cycle $C(A)$ and assume that

$$
\lambda_{1}^{\max }<0<\lambda_{2}^{\min }
$$

Consider any $x \in A$ and note that from (5), for any $\lambda_{2}^{\max }>\epsilon>0$ there is a $T$ such that if $t>T$ then

$$
\left|\Psi_{2}(u, t) v_{2}\right|<\left|v_{2}\right| \exp \left(\left(\lambda_{2}^{\max }+\epsilon\right) t\right)
$$

so that if $T_{12}>T$ we have

$$
\delta<\left|v_{2}\right| \exp \left(\left(\lambda_{2}^{\max }+\epsilon\right) T_{12}\right)
$$


and so

$$
T_{12}>\frac{1}{\lambda_{2}^{\max }+\epsilon} \log \left(\frac{\delta}{\left|v_{2}\right|}\right) .
$$

Thus, if we consider $\nu>0$ such that

$$
\nu<\delta \exp \left(-\left(\lambda_{2}^{\max }+\epsilon\right) T\right)
$$

then for $\left|v_{2}\right|<\nu$ the estimate (5) holds. Similarly, from (5) we can write

$$
\left|\Psi_{1}(u, t) v_{1}\right|<\left|v_{1}\right| \exp \left(\left(\lambda_{1}^{\max }+\epsilon\right) t\right)
$$

and so we have

$$
\left|\Psi_{1}\left(u, T_{12}\right) v_{1}\right|<\delta K_{0}\left|v_{2}\right|^{-\frac{\lambda_{1}^{\max }+\epsilon}{\lambda_{2}^{\max }+\epsilon}}
$$

with $K_{0}$ a positive constant. This implies that there is a $K_{1}$ such that

$$
\left|v_{2}^{\prime}\right|<K_{1}\left|v_{2}\right|^{\frac{-\lambda_{1}^{\max }-\epsilon}{\lambda_{2}^{\lambda_{\max }+\epsilon}}}
$$

for small enough initial $v_{2}$. Similarly using (6) one can obtain the following estimate from below:

$$
\left|v_{2}^{\prime}\right|>K_{1}\left|v_{2}\right|^{\frac{-\lambda_{1}^{\min }+\epsilon}{\lambda_{2}^{\min }-\epsilon}}
$$

Lemma 3.2 Suppose that $\operatorname{Box}(S(A)) \subset U_{4}$. Then the cycle $C(A)$ is asymptotically stable.

Proof :

In this case we have

$$
\lambda_{1}^{\max }<0<\lambda_{2}^{\min }<\lambda_{2}^{\max }<-\lambda_{1}^{\max }
$$

and so we can choose small enough $\epsilon>0$ that the exponent in (11) is strictly greater than unity. Thus we get that all small enough $\left|v_{2}\right|$ converge to zero under iteration of $F$, and a neighbourhood of initial conditions is asymptotic to $C(A)$.

Lemma 3.3 Suppose that $\operatorname{Box}(S(A)) \subset U_{2}$. Then $C(A)$ is normally repelling.

Proof :

Now we have

$$
\lambda_{1}^{\max }<0<-\lambda_{1}^{\max }<-\lambda_{1}^{\min }<\lambda_{2}^{\min }
$$

and by application of (12), all $v_{2}$ close to zero will grow in modulus. Thus no nearby points can be asymptotic to $C$.

\section{Proof :}

(of Theorem 3.1.) This collects the results of Lemmas 3.1, 3.2 and 3.3, after possible re-labelling of $N_{1}$ and $N_{2}$ to interchange $\left(\lambda_{1}, \lambda_{2}\right)$.

The proof of Lemma 3.2 provides us with an upper bound on the asymptotic rates of approach and slowing down: 
Corollary 3.3 Suppose that $B o x(S(A)) \subset U_{4}$; then for all trajectories in $\mathcal{W}^{s}(C(A))$ we have

$$
\max \left(\limsup _{k \rightarrow \infty} R(k), \limsup _{k \rightarrow \infty} r(k)\right) \leq \frac{-\lambda_{1}^{\max }}{\lambda_{2}^{\min }}>1 .
$$

Remark 3.1 It is possible to relax the assumption of a skew product structure if we strengthen the assumptions on the contraction or expansion on $N_{i}$; namely if we assume

$$
\sup _{u \in W}\left\|\exp a_{1}(0,0, u)\right\|<1<\left(\sup _{u \in W}\left\|\exp \left(-a_{2}(0,0, u)\right)\right\|\right)^{-1} .
$$

This has been considered for the case of three coupled cells with $\mathbf{Z}_{3} \dot{+} \mathbf{Z}_{2}^{3}$ symmetry by Field [13]. Note that this condition is dependent on the metric and so is not invariant under conjugacy.

On varying a parameter that preserves the dynamics on $P$ one typically goes through a region where none of the cases in Theorem 3.1 will hold. This is because typically $\left(\lambda_{1}^{n}, \lambda_{2}^{n}\right)$ is in the interior of $S(A)$. We now consider what may happen in this region.

\subsection{Measurable stability}

Even if $A$ is a chaotic saddle it may or may not be contained within another attractor (see e.g. [5]). We first demonstrate that the natural measure dominates behaviour within a neighbourhood of the cycle.

Proposition 3.4 Under the assumptions of Theorem 3.2, the set

$$
\Pi^{-1} \Pi\left(\mathcal{G}^{+}\left(\mu_{n}\right)\right)
$$

has full $\ell_{M}$-measure in some neighbourhood of $C(A)$.

Proof :

Trivially, $\Pi \mathcal{G}^{+}\left(\mu_{n}\right) \supset \mathcal{G}^{+}\left(\mu_{n}\right) \cap P$ and so

$$
\Pi^{-1} \Pi\left(\mathcal{G}^{+}\left(\mu_{n}\right)\right) \supset \Pi^{-1}\left(\mathcal{G}^{+}\left(\mu_{n}\right) \cap P\right) .
$$

Moreover by assumption, $\mathcal{G}^{+}\left(\mu_{n}\right) \cap P$ has full $\ell_{P}$-measure in some neighbourhood of $A$ and it follows that $\Pi^{-1} \Pi\left(\mathcal{G}^{+}\left(\mu_{n}\right)\right)$ has full $\ell_{M}$-measure in a neighbourhood of $\Pi^{-1}(A)$. Note that because $A$ is assumed to be asymptotically stable for the flow restricted to $P$, so by the skew-product structure we have $\Pi\left(\mathcal{W}^{u}(A)\right) \subset A$. Thus $C(A) \subset \mathcal{W}^{u}(A) \subset \Pi^{-1}(A)$ and so $C(A) \subset \Pi^{-1}(A)$; this implies the result.

From here on we consider only trajectories within this full-measure set in some neighbourhood of $C(A)$; these have projections onto $P$ that are generic for $\mu_{n}$. We say a set $A$ has asymptotically full measure near $S \subset \Sigma$ if

$$
\frac{\ell\left(A \cap B_{\delta}(S)\right)}{\ell\left(B_{\delta}(S)\right)} \rightarrow 0
$$

as $\delta \rightarrow 0$, where $\ell($.$) is a Reimannian measure on \Sigma$ and $B_{\delta}(S)$ consists of all points within $\delta$ of a point in $S$. 
Lemma 3.4 If $s_{n} \in U_{6}$ then $A$ is an attractor; if $s_{n} \notin U_{6}$ then it is a chaotic saddle.

\section{Proof :}

This follows on application of [6, Thms. 2.19, 2.20].

Note that if $\operatorname{Box}(S(A)) \cap V=\emptyset$, then all $\lambda_{1}(\mu)$ are of the same sign, as are all $\lambda_{2}(\mu)$. Fix any $0<\epsilon<\min _{i}\left(\left|\lambda_{i}^{n}\right|\right)$ and for any $s$, consider the set of $\left(v_{1}, v_{2}, u\right) \in \Sigma_{1}^{\delta, \gamma}$ such that

$$
\left|v_{2}\right| e^{\left(\lambda_{2}^{n}-\epsilon\right) t}<\left|\Psi_{2}(u, t) v_{2}\right|<\left|v_{2}\right| e^{\left(\lambda_{2}^{n}+\epsilon\right) t}
$$

for $t \geq s$. By (7) as $s \rightarrow \infty$ this set approaches full measure in $\Sigma_{1}^{\delta, \gamma}$. In particular, if we choose

$$
\gamma<\delta \exp \left(-\left(\lambda_{2}^{\max }+\epsilon\right) s\right)
$$

we have $T_{12}<s$ and can assure that (13) holds for $t \geq T_{12}$. Thus, for $\left|\Psi_{2}\left(u, T_{12}\right) v_{2}\right|=$ $\delta$ we have

$$
\frac{1}{\lambda_{2}^{n}+\epsilon} \log \left(\frac{\delta}{\left|v_{2}\right|}\right)<T_{12}<\frac{1}{\lambda_{2}^{n}-\epsilon} \log \left(\frac{\delta}{\left|v_{2}\right|}\right) .
$$

Similarly, by choosing a $\gamma$ (possibly smaller than above) we obtain that

$$
\left|v_{1}\right| e^{\left(\lambda_{1}^{n}-\epsilon\right) t}<\left|\Psi_{1}(u, t) v_{1}\right|<\left|v_{1}\right| e^{\left(\lambda_{1}^{n}+\epsilon\right) t}
$$

for $t=T_{12}$ and so there are constants $K_{i}>0$ such that

$$
K_{0}\left|v_{2}\right|^{\frac{-\lambda_{1}^{s}+\epsilon}{\lambda_{2}^{s}-\epsilon}}<\left|v_{2}^{\prime}\right|<K_{1}\left|v_{2}\right|^{\frac{-\lambda_{1}^{s}-\epsilon}{\lambda_{2}^{s}+\epsilon}}
$$

for a set of $\left(v_{1}, v_{2}, u\right) \in \Sigma_{1}^{\delta, \gamma}$ with asymptotically full measure as $\gamma \rightarrow 0$. We can now state and prove the case for attractivity of $C(A)$.

Lemma 3.5 Suppose that $s_{n} \in \overline{U_{2} \cup U_{4}}$. If $s_{n} \in U_{4}$ then the cycle $C(A)$ is an attractor. Furthermore, if $C(A)$ is an attractor then $s_{n} \in \bar{U}_{4}$. In particular, if $s_{n} \in U_{2}$ then $C(A)$ is not an attractor.

\section{Proof :}

If $\lambda_{1}^{s}<0<\lambda_{2}^{s}<-\lambda_{1}^{s}$, by picking $\epsilon, \delta$ and $\gamma$ small enough, (15) imples there is an asymptotically full measure set of initial conditions in $\Sigma_{i}^{\delta, \gamma}$ that converge to $C(A)$. Conversely, if $s_{n} \in U_{2}$ there is an an asymptotically full measure set of points that are repelled from $C(A)$.

The proof of this result gives a prediction about the mean rates of approach and slowing down in the case that $C(A)$ is an attractor:

Corollary 3.5 If $s_{n} \in U_{4}$ then there is a full $\ell_{M}$-measure set in $\mathcal{W}^{s}(C(A))$ of points whose mean asymptotic rate of approach and slowing down are equal and given by:

$$
R=r=-\frac{\lambda_{1}^{n}}{\lambda_{2}^{n}}
$$


Proof :

By the above argument, for all $\epsilon>0$ there is a full $\ell_{M}$-measure set $\mathcal{W}^{s}\left(C(A), \mu_{n}\right)$ in $\mathcal{W}^{s}(C(A))$ whose trajectories satisfy $\left|R-R_{k}\right|<\epsilon$ for all large enough $k$.

\section{Proof :}

(of Theorem 3.2) This is a collection of Lemmas 3.4 and 3.5, after possible re-ordering of $N_{1}$ and $N_{2}$ to interchange $\left(\lambda_{1}, \lambda_{2}\right)$.

Remark 3.2 The results in this section can be re-interpreted as properties of random dynamical systems [2] because of the assumption of the skew-product structure.

Remark 3.3 An important assumption is that $\operatorname{Box}(S(A)) \cap V$ is empty. If this does not hold, the local dynamics may be much more complicated due to the existence of $\mu \in \operatorname{Erg}(A)$ that are repelling (resp. attracting) in the direction that the natural measure is attracting (resp. repelling). In particular, this will cause the homoclinic orbit to become dirty since it will not be possible to get sections to the flow arbitrarily close to $A$.

\subsection{Local riddling of the basins of attraction}

In the case of $s_{n} \in U_{4}$ but $\operatorname{Box}(S(A))$ intersecting $U_{2}$ one can get local riddling or even riddling of the stable set for $C(A)$.

Theorem 3.6 Suppose that $s_{n} \in U_{4}, \operatorname{Box}(S(A)) \cap V=\emptyset$ and there exists $a \mu \in$ $\operatorname{Erg}(A)$ such that

$$
\left(\nu_{1}, \nu_{2}\right)=\left(\lambda_{1}(\mu), \lambda_{2}(\mu)\right) \in U_{2}
$$

and $\mathcal{G}^{+}(\mu)$ is dense in $A$. Then $C(A)$ is an attractor with a locally riddled basin.

\section{Proof :}

This result follows by methods in Lemma 3.4 and [6, Theorem 2.5] to show that for given fixed $\epsilon>0$ an large enough $s$ the set

$$
\mathcal{Q}(s, \epsilon)=\left\{\left(v_{1}, v_{2}, u\right) \in N_{\delta}:\left|v_{i}\right| e^{\left(\nu_{i}^{s}-\epsilon\right) t}<\left|\Psi_{1}(u, t) v_{i}\right|<\left|v_{i}\right| e^{\left(\nu_{i}^{s}+\epsilon\right) t} \text { for } t>s\right\}
$$

intersects all neighbourhoods of points in $C(A)$ in a set of positive measure. The assumption that $\left(\nu_{1}, \nu_{2}\right) \in U_{2}$ implies that such points exit from a neighbourhood of $A$.

\subsection{Resonance of homoclinic cycle between chaotic sets}

Chow, Deng and Fiedler [9] have studied the problem of unfolding a homoclinic orbit to a fixed point whose real leading eigenvalues have a resonance, i.e. the eigenvalues are of equal magnitude and opposite sign. They observe the creation of a branch of large period orbits in the unfolding. What we observe is a very similar phenomenon, 
but with chaotic attractors created at the bifurcation of the cycle. The scenario we investigate has skew product structure, essentially to simplify the proofs, but we expect the same or similar results if we break the skew product to something in a more general position.

Using results of [9] we can get the following behaviour of fixed point measures in $A$ at resonance. If we assume that $\bar{N}$ is orientable (if $\bar{N}$ is not orientable we get homoclinic doubling) then resonances of fixed point measures $\mu$ supported in $A$ will bifurcate to periodic orbits whose period goes to infinity at the resonance (and whose invariant measures converge weakly to that of the fixed point).

Periodic orbits in $P$ will similarly undergo resonances and lose transverse stability and bifurcate to invariant sets not within $\bar{N}$; presumably these will be quasiperiodic in general. However, even in the skew product case it seems that the bifurcated sets may have complex dynamical behaviour.

We exhibit an example in the following section where one can see a dramatic change in qualitative behaviour as a 'normal parameter' $\nu$ (see [6]) passes through a point $\nu_{0}$ where $s_{n}$ is on the boundary of $U_{4}$ and $U_{2}$. For $\nu<\nu_{0}$ we observe an attracting (but not asymptotically stable) cycle. This is observed to bifurcate to an attractor for $\nu>\nu_{0}$ that is approximately periodic and whose mean period goes to infinity as $\nu \rightarrow \nu_{0}$.

We conjecture that close enough to such a 'homoclinic blowout' bifurcation (cf [22]) there will be a family of attractors $D_{\nu}$ that are 'stuck on' to $N$, i.e. their closures will contain $C(A)$ [5]. Even though the mean period is well defined and finite there may be arbitrarily long quiescent periods. At resonance, the spectra of the ergodic invariant measures supported on the cycle do not lose hyperbolicity; no Lyapunov exponents pass through zero.

\subsection{Resonance and bifurcation}

The results of Theorem 3.2 predict that at a resonance of the natural measure $\mu_{n}$ on $A$

$$
\lambda_{1}\left(\mu_{n}\right)=-\lambda_{2}\left(\mu_{n}\right)
$$

there is a change in qualitative behaviour. Near this resonance we have families of invariant sets that bifurcate from the cycle.

The scenario we get for a normal parameter is as follows: the set $S(A)$ moves across from $U_{4}$ to $U_{2}$, creating families of (unstable) invariant sets at each resonance of a periodic or fixed point measure. We conjecture that the resonance of $\mu_{n}$ has one of two forms which are analogous to the 'hysteretic' and 'non-hysteretic' scenarios of a blowout bifurcation proposed by Ott and Sommerer [22]. These are:

Hard resonance: $\ell_{M}\left(\mathcal{W}^{s}(C(A))\right) \rightarrow 0$ at the resonance. In this case, once the cycle has lost stability, there are no nearby attractors in the sense of natural measures that converge to $\mu_{n}$. This is preceded with riddling of $\mathcal{W}^{s}(C(A))$ by the basin of other attractors.

Soft resonance: $\ell_{M}\left(\mathcal{W}^{s}(C(A))\right) \not \rightarrow 0$ at resonance. There is a family of attractors 
whose natural measures converge to $\mu_{n}$ as we approach the resonance. The support of the measures converges to something much bigger than $C(A)$.

It may be possible to determine the nature of these resonance by looking at the essential basins of attraction of $A$, as seems to be possible for the blowout bifurcation $[7]$.

\section{An example of a homoclinic cycle to a chaotic set}

We consider a modification of a model of Guckenheimer and Holmes [15]. Since we are not considering local problems such as bifurcation from an equilibrium, there is no a priori need to look at low-order polynomial vector fields. Moreover, the model of Guckenheimer and Holmes, being a third order truncation, has degenerate behaviour when the cycle loses stability (this was noted in [15]).

The system has added fifth order terms that to break this degeneracy. It also has skew-product structure to admit a thorough analysis. Although it is an arbitrary model, it is one of the simplest models that can show robust homoclinic cycles to chaotic sets. In particular, because of the splitting (1) it is not possible to find such cycles in systems with dimension less than five.

Consider the function $F: \mathbf{R}^{3} \times \mathbf{R}^{3} \rightarrow \mathbf{R}^{3}$ defined by

$$
\begin{aligned}
& F_{1}(x ; b, c, d)=x_{1}\left(b x_{2}^{2}+c x_{3}^{2}+d x_{2}^{2} x_{3}^{2}\right) \\
& F_{2}(x ; b, c, d)=x_{2}\left(b x_{3}^{2}+c x_{1}^{2}+d x_{3}^{2} x_{1}^{2}\right) \\
& F_{3}(x ; b, c, d)=x_{3}\left(b x_{1}^{2}+c x_{2}^{2}+d x_{1}^{2} x_{2}^{2}\right) .
\end{aligned}
$$

One can project this vector field onto an attracting unit sphere; namely we define

$$
\phi(x ; b, c, d)=F(x ; b, c, d)+\left(1-(x, x)-\frac{(x, F(x ; b, c, d))}{(x, x)}\right) x
$$

where $(x, y)$ is the inner product in $\mathbf{R}^{3}$, and look at the ODE

$$
\dot{x}=\phi(x ; b, c, d) \text {. }
$$

Defining $G(x)=(x, x)$ we note that $\dot{G}=2 G(1-G)$ and so the unit sphere $U=$ $\{x: G(x)=1\}$ is attracting for almost all initial conditions. In particular the flow restricted to $U$ is well-defined. The equation (16) commutes with an action of the group $\Gamma=\mathbf{Z}_{2}^{3} \dot{+} \mathbf{Z}_{3}$ given by cyclic permutation of the axes and reflection in the coordinate planes. If $b, c$ and $d$ are constants, the behaviour of the system (16) as follows:

1. There are fixed points forced by symmetry at $x_{0}=(1,0,0)$ and the group orbit of this point. They have eigenvalues of linearisation $\lambda_{1}=b$ and $\lambda_{2}=c$ tangential to the sphere. These six fixed points are joined by a network of flow-invariant lines corresponding to the intersections of $U$ with the coordinate axes. 
2. If $b c<0$ there is a robust homoclinic cycle connecting the group orbit of $x_{0}$. If additionally $0<(-b / c)^{\operatorname{sgn}(c)}<1$ this cycle is attracting.

3. The case of resonance of the homoclinic cycle is given by $b c<0,|b / c|=1$. There is a periodic orbit branching from the cycle in this case.

4. There are fixed points forced by symmetry at $y_{0}=(\eta, \eta, \eta)$ where $\eta^{2}=\frac{1}{3}$ and the group orbit of this point. These have a complex pair of eigenvalues at $\lambda_{R} \pm i \lambda_{I}$ with

$$
\lambda_{R}=\frac{1}{9}(3 b+3 c+2 d)
$$

and

$$
\lambda_{I}=\sqrt{\frac{1}{2}\left(\left(\frac{2 c}{3}+\frac{2 d}{9}\right)^{2}+\left(\frac{2 b}{3}+\frac{2 d}{9}\right)^{2}\right)+\left(\frac{2 b}{3}-\frac{2 c}{3}\right)^{2}} .
$$

Note that $\lambda_{I}$ is zero only if $b=c=d=0$, and so $y_{0}$ undergoes Hopf bifurcation at $3 b+3 c+2 d=0$.

5. On varying $b, c$ from Hopf bifurcation of $y_{0}$ to the resonance of the homoclinic cycle, there is a path of stable limit cycles connecting one to the other. If $d<0$ they are stable, for $d>0$ they are unstable.

\subsection{Chaotically forcing the model}

We now extend the above model to an O.D.E. $f$ on $M=\mathbf{R}^{6}$ with connections between chaotic sets. This is constructed by parametrically forcing $b, c$ and $d$ with a Rössler system:

$$
\begin{aligned}
& \dot{x}_{i}=f_{i}(x, y) \\
& \dot{y}_{i}=g_{i}(y)
\end{aligned}
$$

where

$$
f_{i}(x, y)=\phi_{i}(x ; b(y), c(y), d(y)), \quad \text { for } i=1,2,3
$$

and

$$
\begin{aligned}
& g_{1}(y)=-\left(y_{2}+y_{3}\right) \\
& g_{2}(y)=y_{1}+0.2 y_{2} \\
& g_{3}(y)=0.2+y_{3}\left(y_{1}-5.7\right)
\end{aligned}
$$

This is a skew-product flow (see e.g. [10]) and the flow on $y$ is a factor, i.e. the projection $\Pi: \mathbf{R}^{6} \rightarrow \mathbf{R}^{3}$ onto the last three components is such that $\dot{y}=g(y)$ is Rössler's system with a chaotic attractor [25]. We shall assume that for the attractor of this flow there exists an absolutely continuous natural measure supported on an asymptotically stable subset $Z \subset \mathbf{R}^{3}$. We consider the functions $b$ and $c$ defined by

$$
b(y)=b_{0}+b_{1} \sin \left(y_{1}\right), \quad c(y)=c_{0}+c_{1} \sin \left(y_{3}\right)
$$

and fix $d$ to be constant. 
The symmetries of the system cause the following subsets of the phase space to be flow-invariant:

$$
\begin{aligned}
P_{1} & =\left\{\left(x_{1}, 0,0\right) \times \mathbf{R}^{3}\right\} \\
P_{2} & =\left\{\left(0, x_{2}, 0\right) \times \mathbf{R}^{3}\right\} \\
P_{3} & =\left\{\left(0,0, x_{3}\right) \times \mathbf{R}^{3}\right\} \\
N_{12} & =\left\{\left(x_{1}, x_{2}, 0\right) \times \mathbf{R}^{3}\right\} \\
N_{23} & =\left\{\left(0, x_{2}, x_{3}\right) \times \mathbf{R}^{3}\right\} \\
N_{13} & =\left\{\left(x_{1}, 0, x_{3}\right) \times \mathbf{R}^{3}\right\}
\end{aligned}
$$

The $P_{i}$ are homeomorphic to $\mathbf{R}^{4}$ while the $N_{i j}$ are homeomorphic to $\mathbf{R}^{5}$.

Lemma 4.1 For $a<0$ there exist chaotic invariant sets $A_{i}$ in $P_{i}(i=1,2,3)$ given by

$$
A_{1}=\{(1,0,0) \times Z\}
$$

etc.

We define the connections between $A_{i}$ and $A_{j}$ to be

$$
C_{i j}=\left\{x: \omega(x) \subset A_{i} \text { and } \alpha(x) \subset A_{j}, i \neq j\right\} .
$$

and the cycle is given by

$$
C=\cup_{i, j} C_{i j}
$$

if it is connected.

By reducing the above system to the orbit space of the group action, one can see the above system in the context of Section 3. In particular, because the system commutes with the action of the group $\Gamma$ it reduces to an ODE on the orbit space $\mathbf{R}^{6} / \Gamma$ i.e. where all points on the same $\Gamma$-orbit are identified. Equivariance means that the flow on the orbit space is well-defined. In this example, all the $P_{i}$ are identified, and all the $N_{i j}$ are identified; the $P_{i}$ can be seen as $P$ and the $N_{i j}$ as $N$ in Figure 1. However, it is equivalent (and easier) to do the computations in the neighbourhoods of a specific $P_{i}$.

Normal Lyapunov Spectrum We calculate the spectrum of normal Lyapunov exponents of $A_{1}$ transverse to $P_{1}$. Note that the invariant subspaces and the skew product structure mean we can write the Jacobian of the flow a point $x=$ $\left(1,0,0, y_{1}, y_{2}, y_{3}\right) \in P_{1}$ in block diagonal form, where $E(y)$ is a $3 \times 3$ matrix.

$$
d_{x} f=\left(\begin{array}{cccc}
-1 & 0 & 0 & 0 \\
0 & c(y) & 0 & 0 \\
0 & 0 & b(y) & 0 \\
0 & 0 & 0 & E(y)
\end{array}\right)
$$

This block diagonal structure means that we can calculate the normal Lyapunov exponents to $A_{1}$ very simply by averaging the derivatives. The transverse Lyapunov exponents for an ergodic invariant measure $\mu$ supported on $A_{1}$ are therefore:

$$
\begin{aligned}
& \lambda_{1}(\mu)=\int_{A_{1}} b(u, v, w) d \mu(u, v, w) \\
& \lambda_{2}(\mu)=\int_{A_{1}} c(u, v, w) d \mu(u, v, w) .
\end{aligned}
$$




\begin{tabular}{|c|cc|}
\hline$c_{1}$ & $\lambda_{1}$ & $\lambda_{2}$ \\
\hline 0.05 & -0.12680 & 0.108146 \\
0.07 & -0.12680 & 0.128146 \\
0.09 & -0.12680 & 0.148146. \\
\hline
\end{tabular}

Table 1: Computed values of the normal Lyapunov exponents calculated for the natural measure on the chaotic invariant set $A$ for three values of $c_{1}$ and $b_{0}=-0.1$, $b_{1}=0.5, c_{1}=0.5, d=-0.1$.

Note that $\bar{\lambda}_{i}(\mu)=\underline{\lambda}_{i}(\mu)$ because the $T_{i}$ are one-dimensional. For a specific pair of functions $b$ and $c$ these Lyapunov exponents form a set

$$
S\left(A_{1}\right)=\left\{\left(\lambda_{1}(\mu), \lambda_{2}(\mu)\right): \mu \in \operatorname{Erg}_{f}\left(A_{1}\right)\right\} .
$$

We assume there exists a natural measure $\mu_{n}$ and consider

$$
s_{n}=\left(\lambda_{1}\left(\mu_{n}\right), \lambda_{2}\left(\mu_{n}\right)\right) .
$$

Typically, $\operatorname{Box}(S)$ will be non-empty and $s_{n}$ in its interior. The simple functional form of $b$ and $c$ assumed in (18) means that we only need to compute one set of averages as follows:

$$
K_{1}(\mu)=\int \sin \left(y_{1}\right) d \mu(y), \quad K_{2}(\mu)=\int \sin \left(y_{3}\right) d \mu(y)
$$

and then

$$
\left(\lambda_{1}(\mu), \lambda_{2}(\mu)\right)=\left(b_{0}+b_{1} K_{1}(\mu), c_{0}+c_{1} K_{2}(\mu)\right) .
$$

In particular, we only need to calculate the ergodic invariant measures once to find $K_{1}$ and $K_{2}$. We fix $b_{0}=-0.1, b_{1}=0.5, c_{1}=0.5, d=-0.1$ and vary $c_{0}$; Figure 4 shows timeseries of $x_{1}(t)$ for the values $c_{0}=0.05, c_{0}=0.07$ and $c_{0}=0.09$. At the same parameter values as those shown in Figure 4, Figure 5 shows the computed values of the normal Lyapunov exponents $\lambda_{i}(\mu)$ near $A_{1}$ for periodic orbits up to period 340 (see Appendix for details of the numerical method). Note that the points scatter over a range of values, and that the interior of the convex hull is non-empty (the special form of $b$ and $c$ causes the footprints to transform affinely with $b_{i}$ and $c_{i}$ ). The normal Lyapunov exponents corresponding to the natural measure are given by

$$
\left(\lambda_{1}, \lambda_{2}\right)=\left(b_{0}-0.05360 b_{1}, c_{0}+0.11629 c_{1}\right)
$$

so that for the given values of $a, b_{0}, b_{1}, c_{0}$ and $d$ and three values of $c_{1}$ considered we can compute normal Lyapunov exponents as in Table 1.

These simulations indicate in agreement with Theorem 3.2, that the cycle is attracting for $c_{0}=0.05$ and repelling in the other two cases. This can be seen from Figure 6 , which shows the 'slowing down' for $c_{0}=0.05$ by plotting the time interval between successive 'proper' crossings of the plane $x_{1}=0.5$ (i.e. crossings corresponding to one return near the fixed point). The average ratio of successive periods 
can be seen to be approximately $1.61=\left(-\lambda_{1} / \lambda_{2}\right)^{3}$, agreeing with the expected slowing down for three approaches to the chaotic saddle. The other cases settle down to a well-defined average period. The resonance occurs at $c_{0}=0.068655$. From the simulations at these parameter values, it appears that the resonance is supercritical, giving rise to a chaotic attractor bifurcating from the cycle.

The example presented above shows a soft resonance as defined in Section 3.5. On changing the sign of the constant $d$ one would expect to see a hard resonance. Before the cycle $C$ has resonance of $\mu_{n}$ we expect to see a locally riddled basin of attraction and by Corollary 3.3, the asymptotic rate of slowing down (if we assume $\lambda_{1}<0<\lambda_{2}$ ) would be given by

$$
r=-\frac{\lambda_{1}\left(\mu_{n}\right)}{\lambda_{2}\left(\mu_{n}\right)} .
$$

As we pass through the resonance, the ratios $r$ and $R$ tend to one. Note that although when $r>1$ we have deviations from $r$, for a full measure subset of $\mathcal{W}^{s}(C(A))$ we expect these deviations will die away as we approach the cycle due to the convergence of the ergodic averages.

\section{Discussion}

Some of the above analysis carries through for semi-flows from evolution equations; in particular the sufficient conditions for being an attractor. The results and conjectures in this paper are consistent with a result of Arnold and Xu Kedai [3]. They prove that a necessary condition for bifurcation of a locally supported invariant measure from a randomly forced fixed point is that a normal Lyapunov exponent passes through zero.

We observe that there can be bifurcation of invariant measures (that are however not locally supported) without a Lyapunov exponent passing through zero.

\subsection{Cycling chaos}

Dellnitz et al. [11] have described a type of dynamical behaviour that is robust in systems with wreath-product symmetries (including the $\mathbf{Z}_{2}^{3} \dot{+} \mathbf{Z}_{3}$ symmetry used in [15] and here). Field [13] has also discussed sufficient conditions for existence of such 'cycling chaos'. We discuss their results in the light of Section 3.

One of the examples they consider is a system of three coupled identical Chua's circuits with cubic nonlinearity; an ODE on $\mathbf{R}^{9}$ :

$$
\begin{aligned}
& \dot{x}_{1}=f\left(x_{1}\right)+\gamma\left|x_{3}\right|^{2} x_{1} \\
& \dot{x}_{2}=f\left(x_{2}\right)+\gamma\left|x_{1}\right|^{2} x_{2} \\
& \dot{x}_{3}=f\left(x_{3}\right)+\gamma\left|x_{2}\right|^{2} x_{3}
\end{aligned}
$$

where $x_{i} \in \mathbf{R}^{3}$ and $\dot{x}_{i}=f\left(x_{i}\right)$ is Chua's circuit equation,

$$
f(u, v, w)=\left(\alpha\left(v-m_{0} u-m_{1} u^{3} / 3\right), u-v+w, \beta v\right) .
$$


with $\alpha=18, \beta=33.136, m_{0}=-0.230769$ and $m_{1}=0.0123077$ in [11, Fig 1]. Each circuit commutes with the action of $\mathbf{Z}_{2}$ on $\mathbf{R}^{3}$ by $(u, v, w) \mapsto(-u,-v,-w)$ and so the origin is a fixed point for Chua's circuit. Thus

$$
A=\left\{(y, 0,0): y \in \mathbf{R}^{3}\right\}
$$

is an invariant subspace containing an attractor $Z$ for the flow restricted to $A$. We can write the derivative of the flow at $(y, 0,0)$ in $3 \times 3$ block-diagonal form:

$$
\left(\begin{array}{ccc}
D f(y) & 0 & 0 \\
0 & D f(0)+\gamma|y|^{2} \operatorname{Id} & 0 \\
0 & 0 & D f(0)
\end{array}\right)
$$

In this setting, $T_{1}$ corresponds to perturbations $(y, z, 0)$ whereas $T_{2}$ corresponds to perturbations $(y, 0, z)$. In terms of the notation of section 3 there are three unstable normal Lyapunov exponents given by the eigenvalues of $D f(0)$ : for the example discussed in [11, Fig. 1] these are

$$
\lambda_{2}=\{-4.1538,3.3333,-1\} .
$$

Note that these are wholly independent of the dynamics on $A$, let alone other invariant measures on $A$. In this direction, there is one unstable direction. The other normal Lyapunov exponents $\lambda_{1}$ are those of the linear cocycle generated by

$$
\dot{z}=\left(D f(0)+\gamma|y(t)|^{2} \mathrm{Id}\right) z, \quad z \in \mathbf{R}^{3}
$$

which depend on the invariant measure via the statistics of $y(t)$, and need to be numerically calculated. Let $\Lambda=\max \lambda_{1}$; from Corollary 3.5 we can expect to see asymptotic slowing down of the cycling for $\Lambda<-3.3333$, but a well-defined average period for $-3.3333<\Lambda<0$ if the trajectory remains close to the cycle. Note that for the timeseries illustrated in their paper, one might guess that [11, Figs 1 and 2] are approximately periodic attractors, while [11, Fig. 3] shows an orbit converging to an attracting homoclinic cycle to a chaotic set. (NB. Simulations have indicated attracting cycles at $\gamma<-0.094$ which appear to pass through resonance to give approximately periodic attractors for $\gamma>-0.094)$.

\subsection{Removing the skew product assumption}

If we examine robust cycles to chaotic sets that arise from chaotic forcing of robust cycles to equilibria, the setting of a skew product is appropriate (see example in Section 4). However, one can expect to see these cycles without any forcing or skew product structure. In particular, they should exist robustly in systems with symmetries where the group acts as a high dimensional representation. For example, the author can see no a priori reason why they should not occur in reaction-diffusion systems on the line with periodic boundary conditions (though they would have to occur away from primary bifurcations).

For the problem of 'riddled basins', Alexander et al. [1] have obtained a general condition for the existence of a positive measure basin of attraction without 
requiring a skew-product assumption. They use Pesin theory $[24,23]$ to construct an absolutely continuous stable foliation of the attractor $\mathcal{W}^{s}\left(A, \mu_{n}\right)$ and prove that this has positive measure. The appropriate way to generalise this construction to cycles to chaotic sets is not clear (although note Remark 3.1).

\subsection{Dirty homoclinic cycles}

There are two ways a homoclinic cycle can be dirty. One way is if $C(A) \not \supset \mathcal{W}^{u}(A)$ in which case there are orbits that directly leave a neighbourhood of $A$ never to return. More subtly, there could be no section to the flow. If there are invariant measures supported on the closure of the $C(A)$ other than $A$ itself, this will obstruct the existence of such a section and there are presumably many more dynamical possibilities. If some invariant measures are transversely attracting and others are transversely repelling, this may lead to nearby invariant sets that need to be included in the analysis before any understanding of the dynamics is possible.

\subsection{Other remarks}

It would inappropriate to finish without mentioning the effects of random perturbations and imperfections that break the invariance of $P$ and $N$. These are very important for understanding potential applications. In particular, one expects that homoclinic orbits will be destroyed and the subtle effects of basin riddling will be

lost. However, what may remain will be effects such as long chaotic transients $[17,30]$.

There will presumably be many new effects if one were to consider robust heteroclinic cycles where the cycle passes near several different invariant sets.

\section{Acknowledgements}

The simulations were performed using the package dstool of Guckenheimer et al. [16]. The author gratefully acknowledges the support of EU HCM grant ERBCHBCT930503 as well as stimulating conversations with Pascal Chossat, Mike Field and Alastair Rucklidge.

\section{Appendix: Numerical method.}

For calculating the ergodic invariant measures and the values of $K_{1}$ and $K_{2}$ from equation (19) an arbitrary initial condition was chosen. The Rössler equations with two additional scalar equations, i.e.

$$
\begin{aligned}
& \dot{y}_{1}=-\left(y_{2}+y_{3}\right) \\
& \dot{y}_{2}=y_{1}+0.2 y_{2} \\
& \dot{y}_{3}=0.2+y_{3}\left(y_{1}-5.7\right) \\
& \dot{u}_{1}=\sin y_{1}
\end{aligned}
$$




$$
\dot{u}_{2}=\sin y_{3}
$$

were integrated with a fourth order Runge-Kutta method (timestep 0.01) and after transients were allowed to decay, approximately 8,400 hits of the surface of section $x_{4}=0, x_{5}<0$ were stored (this corresponds to 50,000 time units). Crossings of the section were found by linear interpolation of the time between two steps bounding

a hit. Periodic points were found simply by scanning through the stored section hits and identifying returns to within 0.002 ; note that the structure of the Rössler attractor is close to a branched one-manifold.

The averages $K_{i}$ over the time-series from $t_{0}$ to $t_{1}$ were approximated by

$$
K_{1}=\frac{u_{1}\left(t_{1}\right)-u_{1}\left(t_{0}\right)}{t_{1}-t_{0}}, \quad K_{2}=\frac{u_{2}\left(t_{1}\right)-u_{2}\left(t_{0}\right)}{t_{1}-t_{0}} .
$$

The average for the natural measure was taken to be the average over the whole time-series; this was monitored to assure that oscillations had decayed after 50, 000 time units. The results were validated by increasing the timestep and the threshold for determining periodic orbits.

\section{References}

[1] J.C. Alexander, I. Kan, J.A. Yorke and Zhiping You. Riddled Basins. Intl. Journal of Bifurcations and Chaos, 2:795-813 (1992).

[2] L. Arnold. Random Dynamical Systems. In: Dynamical systems, Montecatini Terme, 1994, ed R. Johnson. Springer Lecture Notes in Mathematics 1609, Springer-Verlag Berlin (1995).

[3] L. Arnold and Xu Kedai. Invariant measures for random dynamical systems and a necessary condition for stochastic bifurcation from a fixed point. Random \& Computational Dynamics 2:165-192 (1994).

[4] P. Ashwin, J. Buescu and I.N. Stewart. Bubbling of attractors and synchronisation of oscillators. Phys. Lett. A 193:126-139 (1994).

[5] P. Ashwin. Attractors stuck on to invariant subspaces Phys. Lett. A 209:338344 (1995).

[6] P. Ashwin, J. Buescu and I.N. Stewart. From attractor to chaotic saddle: a tale of transverse instability. Nonlinearity 9:703-737 (1996).

[7] P. Ashwin, P.J. Aston and M. Nicol On the unfolding of a blowout bifurcation. Preprint, Department of Mathematical and Computing Sciences, University of Surrey (1996).

[8] P. Ashwin and P. Chossat. Attractors for robust heteroclinic sets with a continuum of connections. Preprint, INLN 96.6 (1996). 
[9] S.-N. Chow, B. Deng and B. Fiedler. Homoclinic bifurcation at resonant eigenvalues. J. Dyn. Diff. Eqns. 2: 177-244 (1990).

[10] I.P. Cornfeld, S.V. Fomin and Ya.G. Sinai. Ergodic Theory. Grundlehren der mathematischen Wissenschaft 245, Springer-Verlag, Berlin (1982).

[11] M. Dellnitz, M. Field, M. Golubitsky, A, Hohmann and J. Ma. Cycling chaos. IEEE Trans. Circuits and Systems-I. 42:821-823 (1995).

[12] M. Field. Geometric methods in bifurcation theory. in Normal forms and homoclinic chaos eds W.F. Langford and W. Nagata, Fields Institute Communications, AMS (1995).

[13] M. Field. Dynamics, Bifurcation and symmetry. Pitmans Research Notes in Mathematics (1996).

[14] A. Gaunersdorfer. Time averages for heteroclinic attractors. SIAM J. Appl. Math. 52:1476-1489 (1992).

[15] J. Guckenheimer and P. Holmes. Structurally stable heteroclinic cycles. Math. Proc. Camb. Phil. Soc. 103:189-192 (1988).

[16] J. Guckenheimer, M.R. Myers, F.J. Wicklin and P.A. Worfolk. Dstool: a dynamical systems toolkit with an interactive graphical interface, user's manual Center for Applied Mathematics, Cornell University (1991).

[17] Y.-C. Lai, C. Grebogi and J.A. Yorke. Riddling bifurcation in chaotic dynamical systems. Phys. Rev. Lett. 77:55-59 (1996).

[18] I. Melbourne, P. Chossat and M. Golubitsky. Heteroclinic cycles involving periodic solutions in mode interactions with $\mathrm{O}(2)$ symmetry. Proc. Roy. Soc. Edinburgh 113A:315-345 (1989).

[19] I. Melbourne An example of a non-asymptotically stable attractor Nonlinearity 4:835-844 (1991).

[20] J. Milnor. On the concept of attractor. Commun. Math. Phys. 99:177-195 (1985); Comments Commun. Math. Phys. 102:517-519 (1985).

[21] V.I. Oseledec. A multiplicative ergodic theorem: Lyapunov characteristic numbers for dynamical systems. Trans. Mosc. Math. Soc. 19:197-231 (1968).

[22] E. Ott, J.C. Sommerer. Blowout bifurcations: the occurrence of riddled basins and on-off intermittency. Phys. Lett. A 188: 39-47 (1994).

[23] M. Pollicott. Lectures on ergodic theory and Pesin theory on compact manifolds. LMS Lecture notes 180, CUP Cambridge (1993).

[24] C. Pugh and M. Shub. Ergodic attractors. Trans. Amer. Math. Soc. 312:1-54 (1989). 
[25] O.E. Rössler Continuous chaos- four prototype equations, Bifurcation theory and applications, Eds O. Gurel and O.E. Rössler, Ann. N. Y. Acad. Sci. 316:376-392 (1979).

[26] A. Rucklidge. pers. comm. (1995).

[27] D. Ruelle. Chaotic evolution and strange attractors, Lezione Lincee, Cambridge University Press (1989).

[28] K. Sigmund. Time averages for unpredictable orbits of deterministic systems. Annals of Operations Research 37:217-228 (1992).

[29] F. Takens. Heteroclinic attractors: time averages and moduli of topological conjugacy. Bol. Soc. Bras. Mat. 25:107-120 (1994).

[30] S.C. Venkataranami, B.R. Hunt and E. Ott. Bubbling transition. Phys Rev E 54:1346-1360 (1996). 


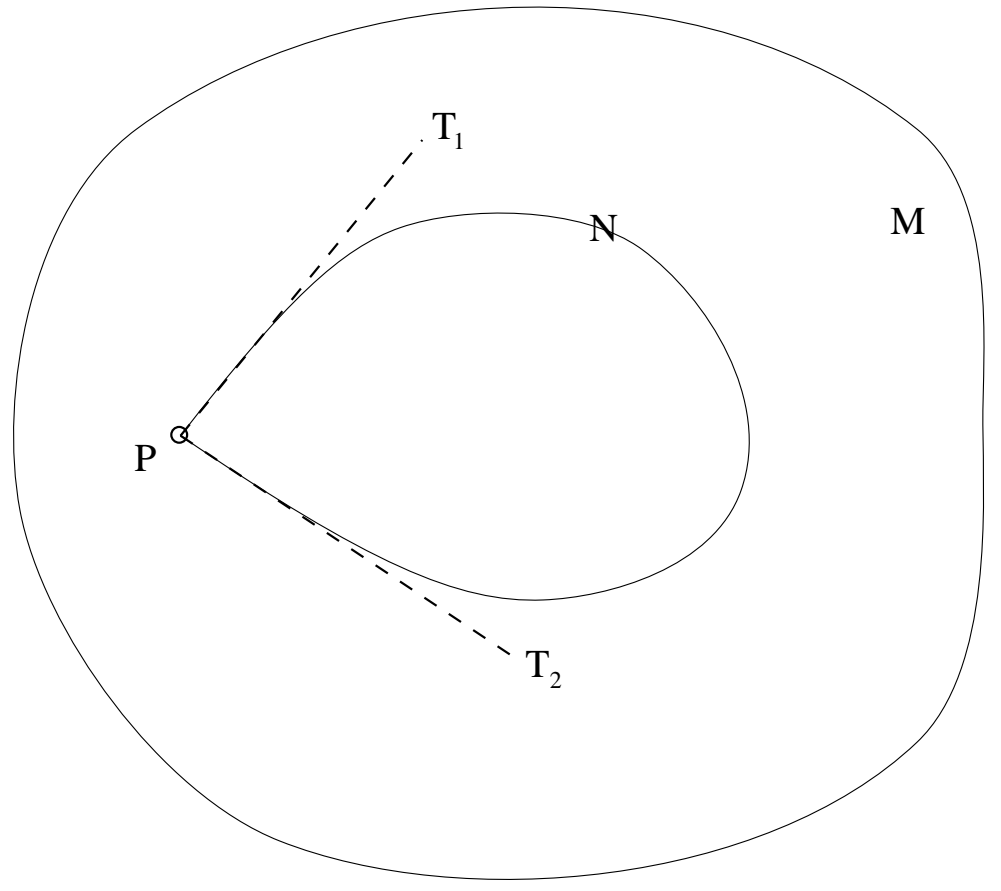

Figure 1: Two subspaces $N$ and $P(P \subset \bar{N})$ are assumed to be invariant under a flow on the phase space $M$. A chaotic attractor exists for the flow restricted to $P$, and robust homoclinic connections may exist contained within $N$. Note that necessary conditions for this are that $3 \leq \operatorname{dim} P$, and also that $\bar{N}$ is not a manifold; it is singular at $P . T_{1}$ and $T_{2}$ represent the two distinct tangent spaces of $N$ at $P$.

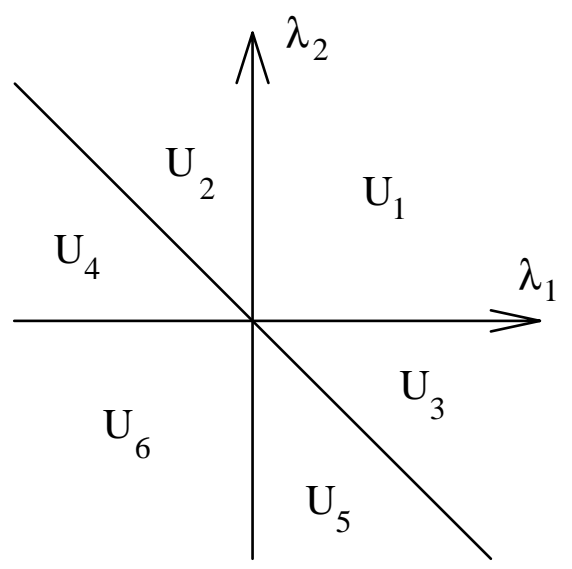

Figure 2: Partition of the $\left(\lambda_{1}, \lambda_{2}\right)$ plane of normal Lyapunov exponents into six regions. The location of $S(A)$ in this plane governs the existence and nature of stability of the cycle between chaotic sets. 


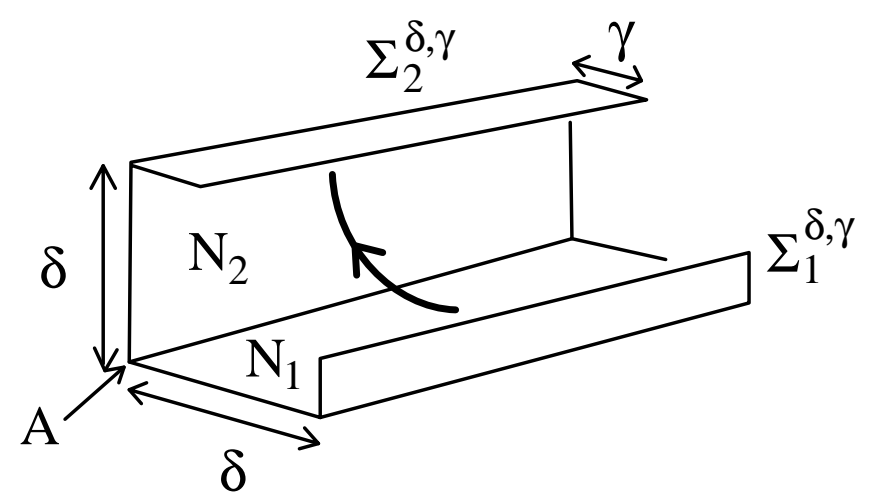

Figure 3: A schematic view of the neighbourhood $N^{\delta}$ and the sections $\Sigma_{i}^{\delta, \gamma}$ near $A$. 


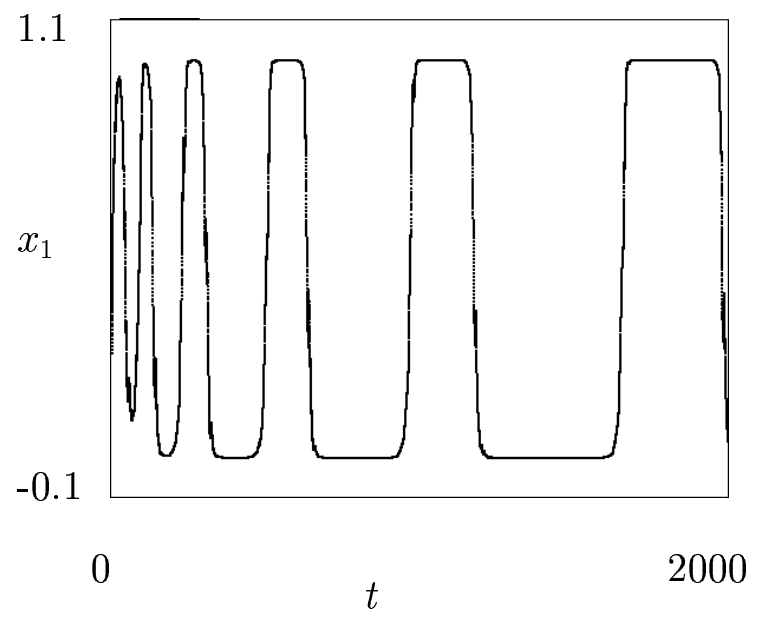

(a)

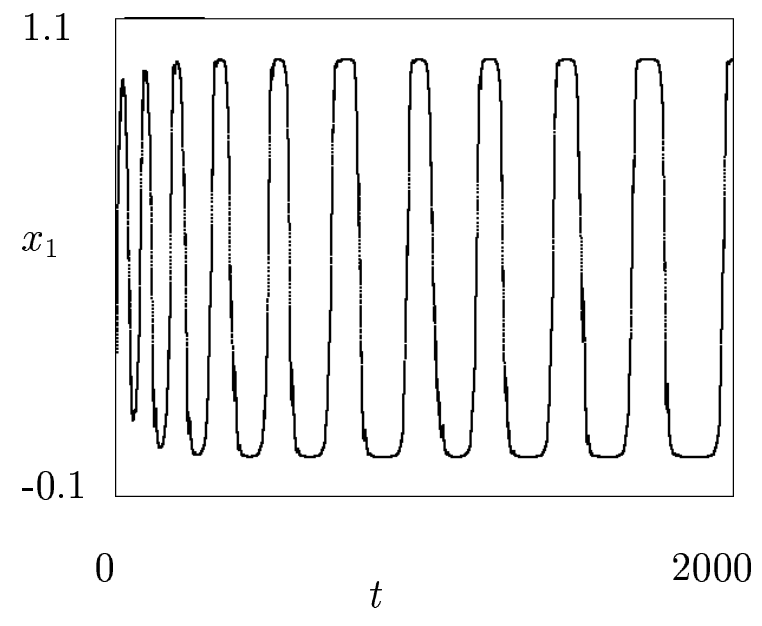

(b)

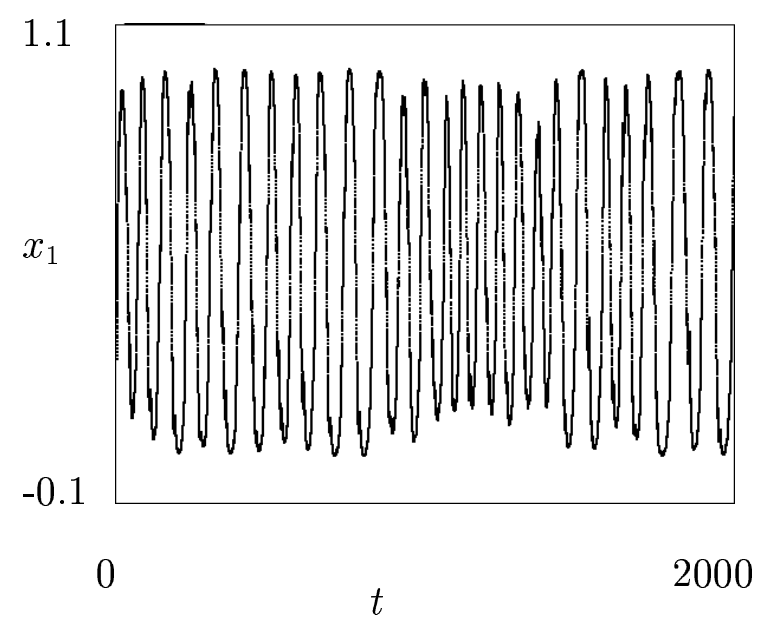

(c)

Figure 4: Timeseries of trajectories of (17) obtained by plotting an observation $x_{1}(t)$ against t. (a) $c_{0}=0.05$, (b) $c_{0}=0.07$, (c) $c_{0}=0.09$. 


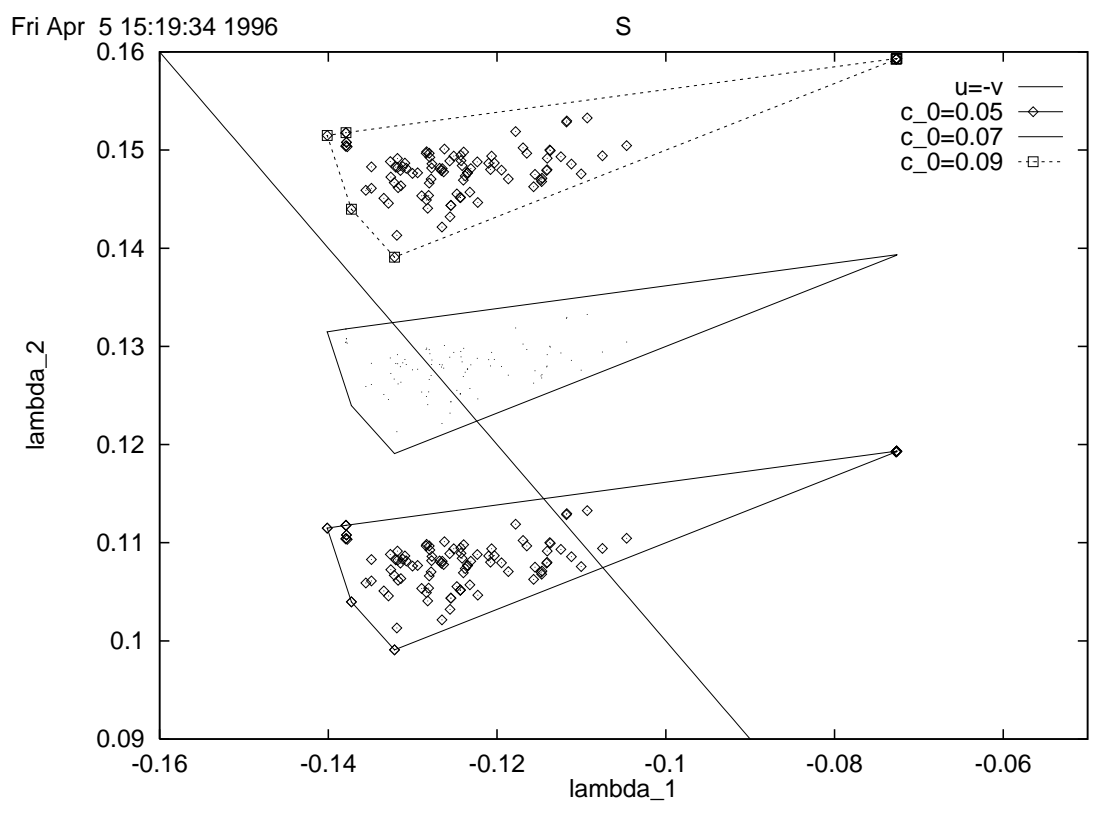

Figure 5: Footprints of normal Lyapunov exponents $\left(\lambda_{1}, \lambda_{2}\right)$ for periodic orbit measures (periods up to 340 Rössler time-units) at the parameter values shown in Figure 4. The cycle is attracting at $c_{0}<0.068655$ and repelling otherwise, as explained by the fact that the normal Lyapunov exponents for the natural measure cross the line $\lambda_{1}=-\lambda_{2}$ at this parameter value. 


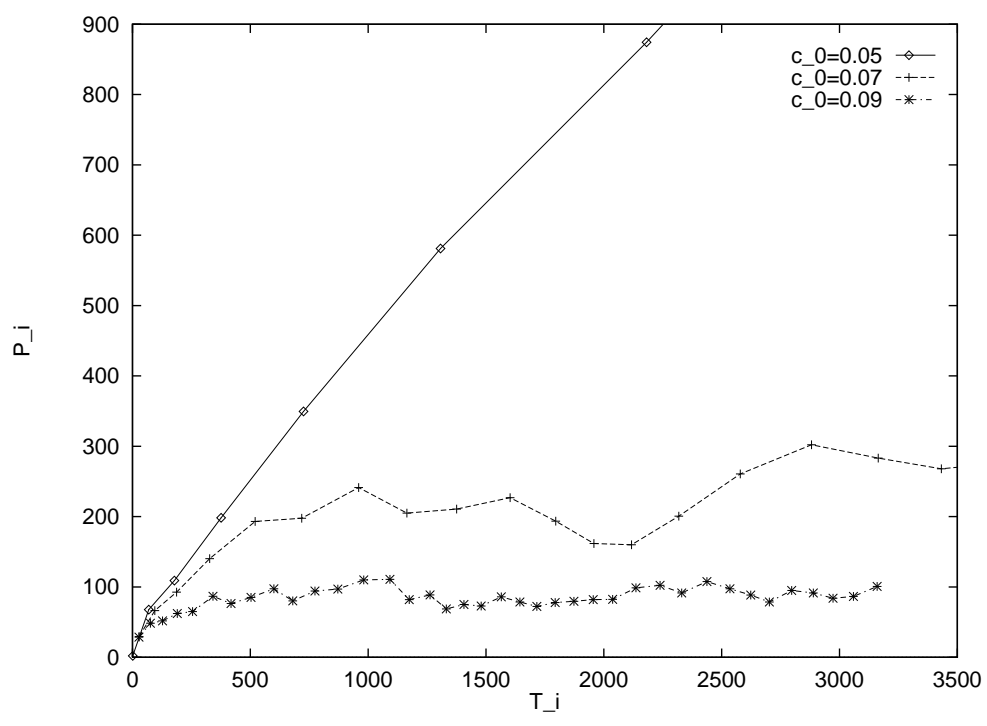

(a)

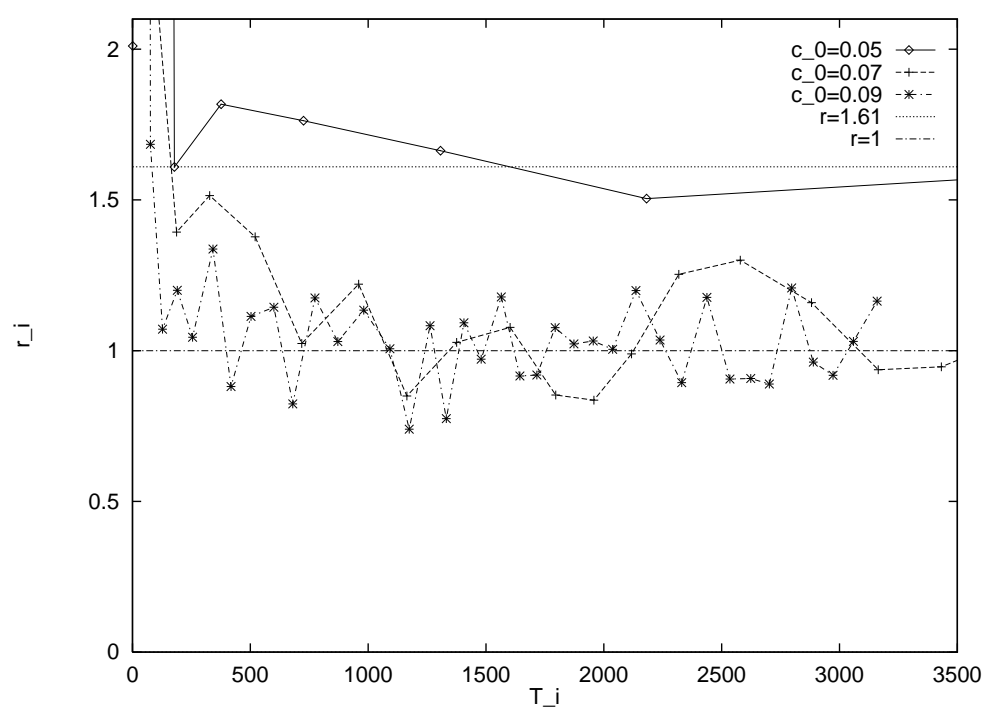

(b)

Figure 6: Slowing down of cycles at the same parameter values as shown in Figure 4. (a) shows period between successive crossings of $x_{1}=0.5$. Note the slowing down for $c_{0}=0.05$, while the other two saturate at some average period. This is clearer in (b) which shows the ratio of successive periods; again, for $c_{0}=0.05$ the average settles down to that expected for three approaches to the chaotic saddle while the others converge to an average of 1.0 (implying approximate periodicity). 